\title{
On Synthetic Measurements of the Turbulent Magnetic Field Property in Young Supernova Remnant
}

\section{Jiro Shimoda*†}

Department of Physics and Mathematics, Aoyama-Gakuin University, Sagamihara, Kanagawa 252-5258, Japan

E-mail: s-jirodphys.aoyama.ac.jp

\section{A. Lazarian}

Department of Astronomy, University of Wisconsin, Madison, WI, USA

\section{Tsuyoshi Inoue}

Department of Physics, Graduate School of Science, Nagoya University, Furo-cho, Chikusa-ku, Nagoya 464-8602, Japan

\begin{abstract}
We present a simple model to show that the spectral slopes of large-scale turbulent magnetic fields in young supernova remnants (SNRs) can be derived from spatial correlation analysis of the polarized synchrotron intensities. The statistical nature of the polarized synchrotron emission is spatially variable owing to the shell-like geometry of the SNR. Setting a synthetic shell that contains two types of turbulent magnetic fields, we analyze the spatial correlations between polarized synchrotron signals from the shell. We find that the correlations that are evaluated at points along the concentric circular-arc of the shell projected onto the sky can recover the spectral slope of given turbulent magnetic fields.
\end{abstract}

35th International Cosmic Ray Conference - ICRC2017

10-20 July, 2017

Bexco, Busan, Korea

* Speaker.

${ }^{\dagger}$ A footnote may follow. 


\section{Introduction}

Galactic cosmic-rays (CRs), which inhabit the energy range below the so-called Knee energy $\left(10^{15.5} \mathrm{eV}\right.$; e.g., [ [8]]), are believed to be accelerated by diffusive shock acceleration (DSA; [W, []]) in supernova remnants (SNRs). Although the magnetic field plays an essential role in the DSA by scattering accelerated particles, properties of magnetic field are remain one of the most unsettled issues in the SNRs, and essential properties of CRs, such as the power-law index of their energy spectra and their maximum energies realized in the SNRs, remain unclear.

Polarized radio synchrotron observations can play a crucial role, in providing valuable information on the SNR magnetic field. Lazarian \& Pogosyan (2012,2016; [Q, [0]) recently suggested that the properties of MHD turbulence with respect to the spectral slope of the magnetic field can be studied by analyzing the fluctuation in polarized synchrotron emissions in the ISM. Zhang et al. (2016) and Lee et al. (2016) numerically confirmed this suggestion for a synthetically reproduced turbulent magnetic field in the cubic domain, however, the SNR has a spherical, shell-like geometry, which affects the observed polarization characteristics. Moreover, there can be multiple types of magnetic field fluctuations driven by different mechanisms, as discussed above. In this study, we analyze a turbulent spherical shell model, which contains two statistically independent magnetic field fluctuations, to demonstrate that the spectral slope of the turbulent magnetic field can be measured by future observations of young SNRs. As typical Very Long Baseline Interferometer radio observations with an angular resolution of $\Delta x \leq 0.01 \mathrm{pc}\left(\frac{\Delta \theta}{11 \operatorname{arcsec}}\right)\left(\frac{d}{1 \mathrm{kpc}}\right)$ can resolve the length scale of the gyroradius of Knee energy CRs, $r_{\mathrm{g}} \sim 1 \mathrm{pc}\left(\frac{E}{\mathrm{PeV}}\right)\left(\frac{B}{10 \mu \mathrm{G}}\right)^{-1}$, we emphasize that we can measure the spectrum of magnetic field fluctuations that resonate with CRs around Knee energy.

\section{Statistical description of polarized synchrotron emission induced by a turbulent magnetic field}

In this paper, we use a statistical description of turbulence as a widely accepted method for characterizing many of its essential properties (e.g., []]). One of the simplest descriptions of the spatial variations of a physical variable, $f(\boldsymbol{r})$, is the so-called correlation function

$$
\begin{aligned}
& \xi(\boldsymbol{l})=\frac{\int d^{3} \boldsymbol{r} f(\boldsymbol{r}) f^{*}(\boldsymbol{r}+\boldsymbol{l})}{\int d^{3} \boldsymbol{r}} \\
& \equiv\left\langle f(\boldsymbol{r}) f^{*}(\boldsymbol{r}+\boldsymbol{l})\right\rangle_{\boldsymbol{r}},
\end{aligned}
$$

where $\langle\ldots\rangle_{\boldsymbol{r}}$ indicates the average over the entire volume of interest. If we consider incompressible and isotropic turbulence, the correlation function of the fluid velocity should show Kolmogorov scaling, $\xi \propto l^{2 / 3}$, in the inertial range. In this case, the Fourier component of $\xi, P_{k}(\boldsymbol{k}) \propto k^{-11 / 3}$, indicates that the kinetic energy spectrum of turbulence, $E_{k}(k) \approx P_{k} k^{2}$, has Kolmogorov scaling $k^{-5 / 3}$. More generally for the scaling relation, when the correlation function of $f(\boldsymbol{r})$ can be written as $\xi \approx l^{m}$ at a finite interval of $l$ and $f(\boldsymbol{r})$ has isotropic fluctuations on a finite scale, the Fourier component of $\xi, P_{k}$, and the Fourier power spectrum, $E_{k} \approx P_{k} k^{2}$, can be approximately written as

$$
\begin{aligned}
& P_{k}(\boldsymbol{k}) \propto k^{-m-3}, \\
& E_{k}(k) \propto k^{-m-1},
\end{aligned}
$$


respectively, from dimensional analysis.

Using the above descriptions, we can statistically describe the polarized synchrotron emission induced by the turbulent magnetic field.

\subsection{Basic definition of synchrotron polarization}

We define the observed synchrotron polarization intensity following Burn (1966) and Lazarian \& Pogosyan (2016),

$$
P\left(\boldsymbol{R}, \lambda^{2}\right)=\int_{0}^{L} d z P_{i}(\boldsymbol{R}, z) e^{2 i \lambda^{2} \Phi(\boldsymbol{R}, z)}
$$

where $P_{i}$ is the intrinsic polarized synchrotron intensity density, $\boldsymbol{R}$ marks the two-dimensional position of the source on the sky, $z$ is the direction of the line of sight, $L$ is the extent of the source along the line of sight, and $\lambda$ is the wavelength of emission. The Faraday rotation measure (RM) is given by

$$
\Phi(\boldsymbol{R}, z)=0.81 \int_{0}^{z} d z^{\prime} n_{e}\left(\boldsymbol{R}, z^{\prime}\right) B_{z}\left(\boldsymbol{R}, z^{\prime}\right) \operatorname{rad~m}^{-2},
$$

where $n_{e}$ is the thermal electron density in units of $\mathrm{cm}^{-3}, B_{z}$ is the magnetic field component parallel to the line of sight in $\mu \mathrm{G}$, and $z$ is the line of sight distance in parsecs. (e.g., [焑]).

\subsection{Statistical description of observed synchrotron polarization}

In this section, following Lazarian \& Pogosyan (2016), we consider the following correlation function of synchrotron polarization intensity,

$$
\begin{aligned}
\xi_{p}\left(\Delta \boldsymbol{R}, \lambda^{2}\right) & \equiv\left\langle P\left(\boldsymbol{R}, \lambda^{2}\right) P^{*}\left(\boldsymbol{R}+\Delta \boldsymbol{R}, \lambda^{2}\right)\right\rangle_{\boldsymbol{R}} \\
& =\frac{\int P\left(\boldsymbol{R}, \lambda^{2}\right) P^{*}\left(\boldsymbol{R}+\Delta \boldsymbol{R}, \lambda^{2}\right) d^{2} \boldsymbol{R}}{\int d^{2} \boldsymbol{R}} \\
& =\int_{0}^{L_{1}} d z_{1} \int_{0}^{L_{2}} d z_{2}\left\langle P_{i}\left(\boldsymbol{R}, z_{1}\right) P_{i}^{*}\left(\boldsymbol{R}+\Delta \boldsymbol{R}, z_{2}\right)\right. \\
& \left.\times e^{-2 i \lambda^{2}\left(\Phi\left(\boldsymbol{R}, z_{1}\right)-\Phi\left(\boldsymbol{R}+\Delta \boldsymbol{R}, z_{2}\right)\right)}\right\rangle_{\boldsymbol{R}},
\end{aligned}
$$

where we interchange the order of the integrations concerned the average of the correlation, $\langle\ldots\rangle_{\boldsymbol{R}}$, and the projection along the line of sight, $\int(\ldots) d z_{1} d z_{2}$. For simplicity, we neglect the Faraday rotation effect so that $\lambda^{2} \Phi \ll 1$. Therefore, the observed correlation is simply written as

$$
\xi_{p}(\Delta \boldsymbol{R}) \approx=\int_{0}^{L_{1}} d z_{1} \int_{0}^{L_{2}} d z_{2}\left\langle P_{i}\left(\boldsymbol{R}, z_{1}\right) P_{i}^{*}\left(\boldsymbol{R}+\boldsymbol{\Delta} \boldsymbol{R}, z_{2}\right)\right\rangle_{\boldsymbol{R}},
$$

Note that the polarized synchrotron intensity emitted by CR electrons depends on $B_{n}{ }^{\gamma}$, where $B_{n}$ is the magnetic field component perpendicular to the line of sight and $\gamma=(p+1) / 2$ ( $p$ is the power-law index of $\mathrm{CR}$ electron energy distribution, $\left.\mathscr{N}_{\mathrm{CR}}\left(E_{\mathrm{CR}}\right) \propto E_{\mathrm{CR}}{ }^{-p}\right)$. Therefore, the expected correlation of synchrotron intensity is $\xi_{i}^{\prime}(\boldsymbol{l})=\left\langle\boldsymbol{B}_{n}(\boldsymbol{r})^{\gamma} \boldsymbol{B}_{n}(\boldsymbol{r}+\boldsymbol{l})^{\gamma}\right\rangle_{\boldsymbol{r}}$. Lazarian \& Pogosyan (2012) showed that

$$
\begin{aligned}
& \xi_{i}^{\prime}(\boldsymbol{l})=\left\langle B_{n}(\boldsymbol{r})^{\gamma} B_{n}^{*}(\boldsymbol{r}+\boldsymbol{l})^{\gamma}\right\rangle_{\boldsymbol{r}} \\
\approx & \mathscr{P}(\boldsymbol{\gamma})\left\langle B_{n}(\boldsymbol{r})^{2} B_{n}(\boldsymbol{r}+\boldsymbol{l})^{2}\right\rangle_{\boldsymbol{r}},
\end{aligned}
$$


where

$$
\mathscr{P}(\gamma)=\frac{\left\langle B_{n}(\boldsymbol{r})^{\gamma} B_{n}^{*}(\boldsymbol{r})^{\gamma}\right\rangle_{\boldsymbol{r}}-\left\langle B_{n}(\boldsymbol{r})^{\gamma}\right\rangle_{\boldsymbol{r}}^{2}}{\left\langle B_{n}(\boldsymbol{r})^{2} B_{n}^{*}(\boldsymbol{r})^{2}\right\rangle_{\boldsymbol{r}}-\left\langle B_{n}(\boldsymbol{r})^{2}\right\rangle_{\boldsymbol{r}}^{2}}
$$

for several $\gamma$ with a power-law correlation function of $B_{n}$. For the range $1.2 \leq \gamma \leq 3$, they reported that the maximum deviation of the normalized $\xi_{i}^{\prime}$ represented in Eq. (2.8) as one of the $\left.\xi_{i}^{\prime}\right|_{\gamma=2}$ is limited to $3 \%$, with $\xi_{i}^{\prime}$ showing the scaling of the magnetic field correlation function. This argument is numerically confirmed by Lee et al. (2016), who derived the Fourier power spectrum from the correlation of the observed synchrotron polarization intensity emitted from a synthetically reproduced turbulent magnetic field for the parameter range $1.5 \leq \gamma \leq 4$, which recovers the spectral index of the given turbulent magnetic field. For SNRs, the observed power-law spectrum in the frequency of the radio synchrotron intensity, $d P / d \lambda^{-1} \propto \lambda^{\alpha}$, typically shows the following spectral slope: $\alpha=(p-1) / 2 \approx 0.6$, that indicates $\gamma \approx 1.4$ (e.g., [四). Thus, in the following section, we assume that the scaling of $\xi_{i}$ is identical to that of a turbulent magnetic field correlation by assuming that there is no correlation between the CR electron number density and the magnetic field fluctuation.

\section{Modeling of an SNR's turbulent shell}

To obtain the correlation of the observed synchrotron polarization intensity in Eq. (2.7), we construct a model of SNR's turbulent emission region. For young SNRs, in particular, the geometry can be represented as a spherical shell (e.g., [지, 미, [4]]).

\subsection{Model description}

We consider a spherical shell with an outer radius of unity and thickness of $w$ (hereafter, all spatial scales are normalized to the outer radius). We set the range of the radial coordinate $r$ as $(1-w)<r<1$.

Because the emission region is shell-like, its extent along the line of sight, $L$, varies spatially with $R$ as

$$
L(R)=2 \sqrt{1-R^{2}} \quad(1-w<R<1) .
$$

In what follows, the turbulent magnetic field is assumed to be isotropic, and its correlation function defined in the inner region of the shell is assumed to be

$$
\begin{aligned}
\xi_{\mathrm{M}}(\boldsymbol{l}) & =\left\langle B(\boldsymbol{r}) B^{*}(\boldsymbol{r}+\boldsymbol{l})\right\rangle_{\boldsymbol{r}} \\
& =\bar{B}^{2}+\sigma_{\mathrm{M}}{ }^{2}\left(\frac{l}{l_{0}}\right)^{m}
\end{aligned}
$$

where $\bar{B}$ is the mean magnetic field strength and $\sigma_{\mathrm{M}}^{2}$ is the variance of the magnetic field strength. Following the discussion in Sect. 凤, the Fourier component of the turbulent magnetic field and its energy spectrum follow the relations as

$$
\begin{aligned}
& P_{k}(\boldsymbol{k}) \propto \sigma_{\mathrm{M}}^{2} k^{n-2} \\
& U_{k}(k) \sim P_{k} k^{2} \propto k^{n},
\end{aligned}
$$


for $2 \pi / l_{0} \leq k \leq k_{\max }$, where

$$
n=-m-1
$$

is the spectral index of the energy spectrum of turbulent magnetic field, $U_{k}$. Following the discussion in Sect. 2.2, we assume that the correlation of the polarized synchrotron intensity, $\xi_{i}$, is identical to the magnetic field correlation.

We assume the following correlations of the intrinsic polarized synchrotron intensity in the emission region as

$$
\xi_{i}\left(\Delta \boldsymbol{R}, z_{1}, z_{2}\right)=\bar{P}_{i}^{2}+\sigma^{2}\left(\frac{l}{l_{0}}\right)^{m}
$$

where $\bar{P}_{i}$ is the mean intrinsic polarized synchrotron intensity over the entire volume of the shell. The variance of the polarized synchrotron intensity is assumed to be $\sigma^{2}=\sigma_{\mathrm{M}}{ }^{2}$, and the index is $m=-n-1$.

\subsection{Model parameters}

Radio synchrotron observations by Dickel et al. (1991) and Reynoso et al. (2013) suggest that the width of the emitting region is $10-30 \%$ of the SNR radius, and therefore, we set the thickness of the shell as $w=0.3$. The normalization constant in Eq. (B.6), $l_{0}$, is set to unity.

Reynoso et al. (2013) observed a highly inhomogeneous polarized intensity image in the young SNR. Thus, we assume that the variance component of the intrinsic polarization intensity dominates the mean component, i.e., $\sigma^{2} \gg \bar{P}_{i}^{2}$.

We set the scaling of the magnetic field correlation function to $m=2 / 3$ that gives Kolmogorovlike scaling, $U_{k} \propto k^{-5 / 3}$.

\section{Results of the correlation analysis of the polarized synchrotron emission}

In this section, we show the spatial dependence of the observed correlation.

Even though we have assumed the simple geometry of a spherical shell, Eqs. (B.6), (B.]), and (2.7) still imply a complicated profile for the observed correlation. If we consider the intrinsic correlation as $\xi_{i}=\left(l / l_{0}\right)^{2}$, the observed correlation can be derived analytically as

$$
\left.\xi_{p}(\Delta \boldsymbol{R})\right|_{m=2}=\left(\frac{\Delta \boldsymbol{R}}{l_{0}}\right)^{2} L_{1} L_{2}+\frac{1}{48} \frac{L_{1}^{4}+3 L_{1}^{2} L_{2}^{2}+L_{2}^{4}}{l_{0}^{2}},
$$

where $L_{1}=L(|\boldsymbol{R}|), L_{2}=L(|\boldsymbol{R}+\Delta \boldsymbol{R}|)$. The result indicates the difficulty of removing the geometrical effect from the observed correlation, and the fourth term of the right hand side of Eq. (4.]) shows the different scaling from $m=2$ as $L_{2}{ }^{4}=\left(1-\left|\boldsymbol{R}_{\mathbf{1}}+\Delta \boldsymbol{R}\right|^{2}\right)^{2} \propto|\Delta \boldsymbol{R}|^{4}$.

However, Eq. (4.]) provides a hopeful expression in which in selecting the separated sky positions along the concentric circular-arc of the shell projected onto the sky, the observed correlation depends only on the radius of the arc as

$$
\left.\xi_{p}\right|_{m=2}=\left(\frac{\Delta \boldsymbol{R}}{l_{0}}\right)^{2} L^{2}+\frac{L^{4}}{8 l_{0}{ }^{2}}
$$


That is, the geometrical effect of the shell can be suppressed, enabling the observed correlation to recover the scaling of the turbulent magnetic field. Indeed, when $|\Delta \boldsymbol{R}| \gg L$, the observed correlation is derived from Eq. (2.]T) as

$$
\xi_{p}(\Delta \boldsymbol{R}) \approx|\Delta \boldsymbol{R}|^{m} L^{2}
$$

We show the results of the observed correlations on the concentric circular-arc of radius $R_{1}$ . Figure $\square$ represents the observed correlations at several $R_{1}$, with the curves showing the results $R_{1}=0.97, \ldots, 0.73$ from top to bottom. The observed correlations are expected to recover the scaling of the given turbulent magnetic field for larger $|\Delta \boldsymbol{R}|$.

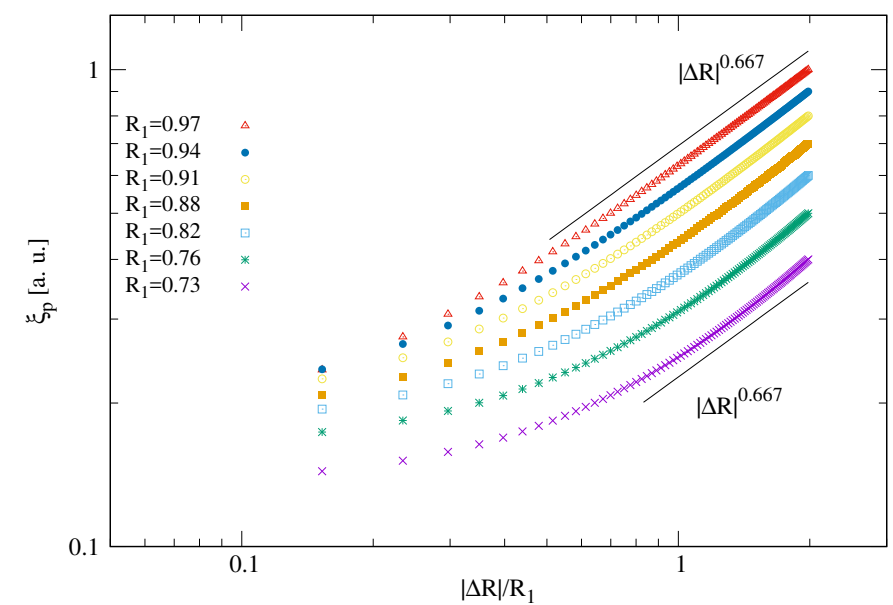

Figure 1: The observed correlation evaluated at the points onto the concentric circular-arc of the radius $R_{1}$ of the shell projected on the sky. The different colors, from red to magenta, represent different arc radii $\left(R_{1}=0.97, \ldots, 0.73\right)$.

\section{Summary}

Using the formulation in Eq. ([2.]), we studied the spatial correlation of short-wavelength polarized synchrotron emissions from a spherical shell. These correlations were evaluated at the points on the concentric circular-arc of the shell projected onto the sky at which its geometrical effect can be suppressed, and it was shown that the correlations on the arc recovered the spectral slope of the given turbulent magnetic field. It was found that taking the correlation with $\Delta R \gg L$, produced an expected observed correlation of $\xi_{p} \approx|\Delta \boldsymbol{R}|^{m}$, where $L$ is the thickness of the turbulent region along the line of sight and $m$ is the spectral index of the turbulent magnetic field. This indicates that the scaling of the turbulent magnetic field is detectable.

\section{References}

[1] A. R. Bell, The acceleration of cosmic rays in shock fronts - I, MNRAS 182 (1978) 
[2] D. Biskamp, Magnetohydrodynamic Turbulence, ed. D. Biskamp (Cambridge: Cambridge Univ. Press), XI (2003)

[3] R. D. Blandford, \& J. P. Ostriker, Particle Acceleration by Astrophysical Shocks, ApJ 221 (1978)

[4] M. A. Brentjens, \& A. de Bruyn, Faraday rotation measure synthesis, A\&A 441 (2005)

[5] B. J. Burn, On The Depolarization of Discrete Radio Sources by Faraday Dispersion, MNRAS 133 (1966)

[6] J. R. Dickel, W. J. M. Breugel, \& R.G. Strom, Radio Structure of The Remnant of Tycho's Supernova (SN 1572) AJ 101 (1991)

[7] D. A. Green A revised Galactic supernova remnant catalogue BASI 37 (2009)

[8] A. M. Hillas, in proceedings of 17th International Cosmic Ray Conference (1981)

[9] A. Lazarian, \& D. Pogosyan, Statistical Description of Synchrotron Intensity Fluctuations: Studies of Astrophysical Magnetic Turbulence ApJ 747 (2012)

[10] A. Lazarian, \& D. Pogosyan, Spectrum and Anisotropy of Turbulence from Multi-Frequency Measurement of Synchrotron Polarization ApJ 818 (2016)

[11] H. Lee, A. Lazarian, \& J. Cho, Polarization Studies of Magnetic Turbulence with An Interferometer ApJ 831 (2016)

[12] E. M. Reynoso, J. P. Hughes, \& D. A. Moffett, On The Radio Polarization Signature of Efficient and Inefficient Particle Acceleration in Supernova Remnant SN 1006 AJ 145 (2013)

[13] J. S. Warren, J. P. Hughes, C. Badenes, et al. Cosmic-Ray Acceleration at The Forward Shock in Tycho's Supernova Remnant: Evidence From CHANDRA X-Ray Observation ApJ 634 (2005)

[14] P. F. Winkler, B. J. Williams, S. P. Reynolds, et al. A High-Resolution and Optical Study of SN 1006: Asymmetric Expansion and Small-Scale Structure in A Type Ia Supernova Remnant ApJ 781 (2014)

[15] J. F. Zhang, A. Lazarian, H. Lee, \& J. Cho, Studying Magnetohydrodynamic Turbulence with Synchrotron Polarization Dispersion ApJ 825 (2016) 